\title{
Parallel View-Dependent Refinement of Progressive Meshes
}

\author{
Liang $\mathrm{Hu}^{*}$ \\ Pedro V. Sander ${ }^{\dagger}$ \\ Hong Kong University of Science and Technology
}

\author{
Hugues Hoppe $e^{\ddagger}$ \\ Microsoft Research
}

\begin{abstract}
We present a scheme for view-dependent level-of-detail control that is implemented entirely on programmable graphics hardware. Our scheme selectively refines and coarsens an arbitrary triangle mesh at the granularity of individual vertices, to create meshes that are highly adapted to dynamic view parameters. Such fine-grain control has previously been demonstrated using sequential CPU algorithms. However, these algorithms involve pointer-based structures with intricate dependencies that cannot be handled efficiently within the restricted framework of GPU parallelism. We show that by introducing new data structures and dependency rules, one can realize fine-grain progressive mesh updates as a sequence of parallel streaming passes over the mesh elements. A major design challenge is that the GPU processes stream elements in isolation. The mesh update algorithm has time complexity proportional to the selectively refined mesh, and moreover can be amortized across several frames. The static data structure is remarkably compact, requiring only $57 \%$ more memory than an indexed triangle list. We demonstrate real-time exploration of complex models with normals and textures.
\end{abstract}

\section{Introduction}

Efficient rendering of complex geometric models has been an active research area for over a decade, with many level-of-detail (LOD) representations offering tradeoffs in fidelity and speed. Intuitively, models that are far away are rendered as coarser meshes. The most challenging setting is a large-scale model that cannot be partitioned into independent parts. Its surface mesh must be adapted in realtime to selectively refine the nearby visible regions. Several solutions to this view-dependent LOD problem have been explored previously, as reviewed in Section 2.

Many early techniques for view-dependent LOD provide fine granularity via vertex splits and edge collapses (Figure 1). These techniques require sequential CPU algorithms to update pointerbased data structures with intricate dependencies, and are therefore difficult to implement efficiently on present system architectures. First, the sequential algorithms cannot benefit from the available GPU parallelism. And second, because the mesh is modified in system memory, a duplicate copy must be transferred to video memory at every frame for rendering. As a consequence, more recent LOD research instead focuses on coarsely partitioning the mesh and storing static buffers in video memory. The sacrifice in LOD granularity is offset by an improvement in frame rate.

Contribution In this paper, we present a framework that allows vertex-level LOD updates to be performed in parallel on the GPU, with all the data residing in video memory. Our approach makes use of the programmable geometry shader introduced in recent GPUs, which is able to process a stream of elements in parallel.

\footnotetext{
*e-mail: nickyhu@ cse.ust.hk

${ }^{\dagger}$ http://www.cse.ust.hk/ psander/

${ }^{\ddagger}$ http://research.microsoft.com/ hoppe/
}

Parallel view-dependent LOD control is a daunting problem. We must perform "surgery" over an entire mesh as a parallel process, to both coarsen and refine it adaptively, while maintaining consistent connectivity, i.e. a watertight surface without holes, T-junctions, or duplicate faces. Moreover, the computational model for GPU streaming has major restrictions. Each stream element is processed in complete isolation from all others, and a streaming pass cannot both read and write the same memory buffer. In light of this, we were initially unsure that a practical solution would be realizable.

Approach overview Previous fine-grain LOD algorithms all use a traditional mesh data structure, in which each triangle face contains references to its 3 neighboring faces. We soon discovered that such a structure poses an obstacle because updating the references in parallel is unwieldy. Face references cannot be read and written in the same pass, and become stale after a single modification. One of our insights is to design new data structures that obviate the need for face neighbor references. In turn, the absence of neighbor information requires new dependency conditions for vertex split and edge collapse operations within the progressive mesh structure (Section 3).

Our runtime representation has two main parts (Section 4). A set of static structures encode a progressive mesh hierarchy for the detailed input mesh. And, a set of dynamic structures encode the active, selectively refined mesh. A unique aspect is that the active mesh is fully specified by a stream of vertices. This stream contains all vertices "above" the active frontier in the vertex hierarchy, and simultaneously identifies both (1) the active vertices, which are on the frontier, and (2) the active faces, which are created by vertices split above the frontier.

We perform a set of parallel streaming passes to update the vertex stream as the view parameters change, and to create an index buffer for rendering (Section 5). All per-frame computations are performed in time proportional to the complexity of the active mesh rather than the fully detailed input mesh. Additionally, we automatically partition this computation across multiple frames to maintain a fixed frame rate (Section 6).

\section{Previous work}

There is vast literature describing different simplification and LOD management strategies, as well as different error metrics and criteria. For a comprehensive overview, including the original CPUbased geometry update methods, refer to Luebke et al. [2002].

Early CPU methods for view-dependent LOD over arbitrary meshes use fine-grain updates based on vertex splits and edge collapses [Xia and Varshney 1996; Hoppe 1997] or octree-based vertex clustering [Luebke and Erikson 1997]. As the GPU has become more powerful, more recent methods typically maintain a fixed set of static buffers in video memory, and switch or geomorph among them [Cignoni et al. 2004; Borgeat et al. 2005; Sander and Mitchell 2005].

An interesting special case is that of terrains, whose vertices lie on regular 2D grids. Such specialized methods are based on both fine-grain mesh updates [Lindstrom et al. 1996; Duchaineau et al. 1997; Lindstrom and Pascucci 2002], and coarse-grain updates [Levenberg 2002; Cignoni et al. 2003]. More recent work renders nested regular grids about the viewer [Losasso and Hoppe 2004]. Our work differs in that it handles the general case of arbitrary meshes. 
Programmable graphics hardware has allowed many surface tessellation approaches to migrate to the GPU, including isosurface extraction [Pascucci 2004; Buatois et al. 2006], subdivison surfaces [Shiue et al. 2005], NURBS patches [Guthe et al. 2005], and procedural detail [Boubekeur and Schlick 2005; Bokeloh and Wand 2006]. Whereas these approaches are typically used to amplify coarse geometry, our refinement framework is designed to exactly reproduce an arbitrary detailed mesh.

Two recent GPU-based LOD techniques are most closely related to our goal of faithfully preserving detail of an arbitrary input mesh. DeCoro and Tatarchuk [2007] present a scheme for simplifying arbitrary meshes using octree-based vertex clustering. This clustering strategy avoids precomputation and storage of a vertex hierarchy, but the resulting approximating meshes are less accurate. Ji et al. [2006] also perform LOD computations on the GPU. Their technique first resamples the input model onto a regular remesh over a polycube map. A vertex shader is used to displace inactive vertices to infinity. One key difference of our work from these two techniques is that we do not require traversing the entire representation (e.g. the fully detailed input mesh) at every frame. Instead, our per-frame computation is only proportional to the (selectively refined) rendered mesh. Our system is the first to perform real-time vertex-grain LOD over arbitrary triangle meshes on the GPU.

\section{Vertex hierarchy and refinement dependencies}

\subsection{Vertex hierarchy}

Our view-dependent LOD algorithm uses the vertex hierarchy of a progressive mesh (PM) [Xia and Varshney 1996; Hoppe 1997]. The construction is based on that of Hoppe [1997], with modifications tailored to our parallel LOD update algorithm.

Given an arbitrary mesh $M^{n}$, a hierarchy is built by applying a prioritized sequence of edge collapses:

$$
M^{n} \underset{s p l_{n-1}}{\stackrel{c o l_{n-1}}{\rightleftharpoons}} \ldots \ldots \frac{c o l_{1}}{\underset{s p l_{1}}{\rightleftharpoons}} M^{1} \underset{s p l_{0}}{\stackrel{c o l_{0}}{\rightleftharpoons}} M^{0}
$$

where $\operatorname{col}_{v}$ is the collapse that creates vertex $v, s p l_{v}$ is the reverse operation that splits $v$, and $M^{0}$ is the base mesh that results after all the collapses. Our method restricts the collapses to half-edge collapses to produce a compact read-only vertex buffer [Kobbelt et al. 1998].

Figure 1 shows a collapse and the split that inverts the operation. The pair of faces $f_{l}^{v}$ and $f_{r}^{v}$, which are adjacent to $v_{t}$ and $v_{u}$, are removed by $\operatorname{col}_{v}$, and are created by $s p l_{v}$. During PM construction, we reorder the 3 vertices of every face in $M^{n}$ such that when performing $\operatorname{col}_{v}$, the vertices in $f_{l}^{v}$ and $f_{r}^{v}$ are $\left\{v_{t}, v_{u}, v_{l}\right\}$ and $\left\{v_{u}, v_{t}, v_{r}\right\}$ respectively.

Figure 2 shows the subtree rooted at $v$ in a vertex hierarchy. The leaves are the vertices in $M^{n}$, and each non-leaf vertex $v$ results from the collapse of its two children $v_{t}$ and $v_{u}$. Since half-edge collapses are used, $v$ has the same attributes (e.g., position, normal) as its left child $v_{t}$ and transitively its leftmost descendant leaf, denoted as $v_{t^{*}}$. Thus we need only store vertex attributes for the leaves, and access them for any vertex $v$ using $v_{t^{*}}$. We let $v^{p}$ denote the parent of $v$, i.e. $\left(v_{t}\right)^{p}=v$. The vertex hierarchy is linearized in memory, with vertices assigned indices in the reverse order that they were collapsed. Thus the leaf vertices are consecutive and last, and can be distinguished from non-leaf vertices solely by their index. The ordering also implies that $v>v^{p}$ for any vertex $v$.

A selectively refined mesh, denoted $M$, corresponds to a frontier of active vertices within the hierarchy, as illustrated in Figure 2. This frontier partitions the (non-leaf) vertices into split and collapsed states.

\subsection{Refinement dependency structure}

To prevent foldovers of the triangles in the mesh, the splits and collapses must adhere to dependency rules. In order to produce

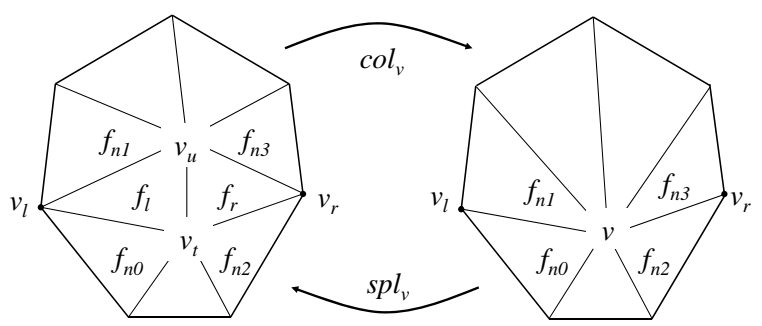

Figure 1: The neighborhood around a split/collapse operation.

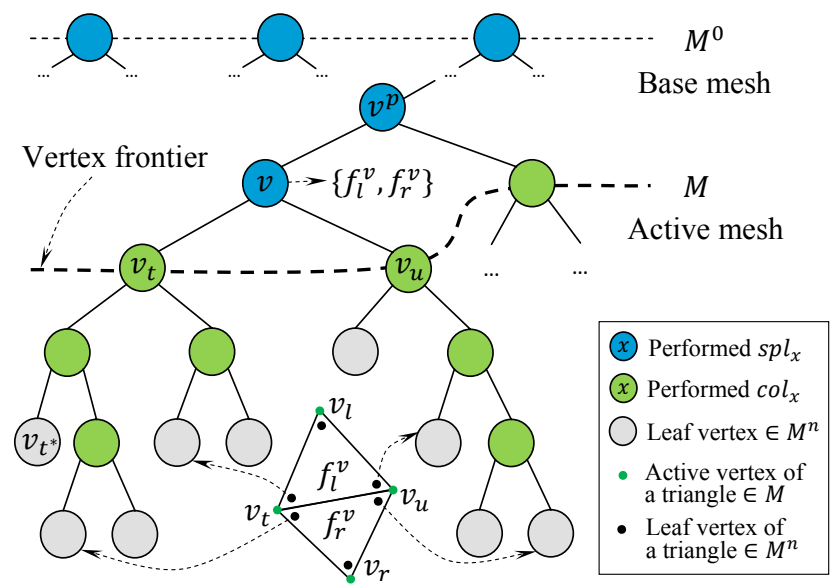

Figure 2: Vertex hierarchy showing a subtree rooted at a vertex $v$. Splitting $v$ generates vertices $v_{t}, v_{u}$ and the pair offaces $\left\{f_{l}^{v}, f_{r}^{v}\right\}$. A selectively refined mesh $M$ corresponds to a frontier of active vertices. All vertices above this frontier, colored blue, have been split, whereas all vertices colored green are in their collapsed state. The leaf vertices of the triangles $f_{l}^{v}$ and $f_{r}^{v}$ are shown with their locations in the hierarchy.

a compact vertex hierarchy and perform efficient runtime tests on the GPU, we introduce a new dependency structure. The explicit rules [Hoppe 1997] incur extra memory, whereas the implicit rules [El-Sana and Varshney 1999] are too restrictive and would require many more unnecessary vertex splits to meet the viewdependent criteria. Additionally, they all require relatively complex runtime tests. Our proposed approach follows the same refinement flexibility as the explicit rules, but with a more compact representation inspired by the implicit rules, and most importantly it is well adapted to GPU stream processing due to its simplicity.

During the PM construction, for each $\mathrm{col}_{v}$, each removed face $f_{l}^{v}$ and $f_{r}^{v}$ is adjacent to two other mesh faces, $\left\{f_{n_{0}}^{v}, f_{n_{1}}^{v}\right\}$ and $\left\{f_{n_{2}}^{v}, f_{n_{3}}^{v}\right\}$ respectively, as shown in Figure 1. At runtime, the explicit rules [Hoppe 1997] check for the presence and adjacency of these four faces in the current selectively refined mesh. Specifically the rules are as follows:

(i) A split $s p l_{v}$ is legal if the faces $\left\{f_{n_{0}}^{v}, f_{n_{1}}^{v}, f_{n_{2}}^{v}, f_{n_{3}}^{v}\right\}$ all exist in the current selectively refined mesh.

(ii) A collapse $\operatorname{col}_{v}$ is legal if $f_{l}^{v}$ is currently adjacent to $\left\{f_{n_{0}}^{v}, f_{n_{1}}^{v}\right\}$ and $f_{r}^{v}$ is currently adjacent to $\left\{f_{n_{2}}^{v}, f_{n_{3}}^{v}\right\}$.

Unfortunately, test (ii) involves maintaining face adjacencies, which is difficult in a parallel algorithm. Our approach is to perform a simpler check that involves storing two vertex indices instead of four face indices. Specifically, we precompute $v_{l \max }=$ $\max \left(c_{v}\left(f_{n_{0}}^{v}\right), c_{v}\left(f_{n_{1}}^{v}\right)\right)$ and $v_{\max }=\max \left(c_{v}\left(f_{n_{2}}^{v}\right), c_{v}\left(f_{n_{3}}^{v}\right)\right)$ where $c_{v}(f)$ is a non-ancestral vertex split that creates $f$. More precisely, $c_{v}(f)$ is the vertex $x$ whose split creates face $f$, unless $f \in M^{0}$ or $x$ is an ancestor of $v$, in which cases $c_{v}(f)=0$. At 
Table 1: Buffers used in runtime selective refinement. Labels $L$ and I denote leaf and non-leaf nodes of the vertex hierarchy. Memory usage is in bytes, where $n$ and $m$ are the number of vertices in the original mesh $M^{n}$ and active mesh $M$ respectively. ${ }^{\dagger}$ Typically the base mesh $M^{0}$ has negligible complexity.

\begin{tabular}{|c|c|c|c|}
\hline BUFFERS & ELEMENTS & CARDINALITY & MEMORY \\
\hline \multicolumn{4}{|l|}{ Static structures } \\
\hline VertexBuffer & $\begin{array}{l}\text { Position } \\
\text { Texcoord } \\
\text { Normal }\end{array}$ & $\mathbf{L}$ & $\begin{array}{l}12 n \\
4 n \\
4 n\end{array}$ \\
\hline VertexTree & $\begin{array}{c}\left\{v_{t}, v_{u}, v_{t^{*}}\right\} \\
v^{p}\end{array}$ & $\begin{array}{l}\text { I } \\
\text { L I }\end{array}$ & $\begin{array}{l}12 n \\
8 n\end{array}$ \\
\hline RefineCriteria & $\left\{\delta_{v}, r_{v}, \sin ^{2} \alpha_{v}\right\}$ & $\mathbf{I}$ & $4 n$ \\
\hline OrigFaces & $\begin{array}{c}\left\{v_{0}, v_{1}, v_{2}\right\} \\
v_{l \max }\left(v_{r \max }\right)\end{array}$ & $M^{n}$ & $\begin{array}{l}18 n \\
6 n\end{array}$ \\
\hline BaseVStream & $v$ & $M^{0}$ & $--^{\dagger}$ \\
\hline BaseFStream & $f$ & $M^{0}$ & $-{ }^{\dagger}$ \\
\hline \multicolumn{4}{|c|}{ Dynamic structures } \\
\hline VertexState & $v_{\text {state }}$ & I & $n$ \\
\hline VertexStream & $v$ & $M$ & $4 m \times 2$ \\
\hline IndexBuffer & $\left\{v_{0}, v_{1}, v_{2}\right\}$ & $M$ & $24 m \times 2$ \\
\hline Total & & & $69 n+56 m$ \\
\hline
\end{tabular}

runtime, given the side vertices $v_{l}$ and $v_{r}$ in $M$, we can check legality as follows:

(I) A split $s p l_{v}$ is legal if $v_{l}>v_{l \max }$ and $v_{r}>v_{\max }$.

(II) A collapse $\operatorname{col}_{v}$ is legal if $\left(v_{l}\right)^{p}<v$ and $\left(v_{r}\right)^{p}<v$.

Our dependency rules conform to the explicit rules, which is proven in the appendix.

\section{Data structures}

Our framework maintains a static VertexBuffer holding the vertex attributes, and a dynamically updated IndexBuffer holding 3 vertex indices per triangle face. These structures are used to render the selectively refined mesh using a generic vertex shader. To maintain the IndexBuffer, several static and dynamic data structures are required. These data structures, which are listed in detail in Table 1, are stored on the GPU using sequential buffers. Note that we keep a double-buffered IndexBuffer to allow asynchronous update of one index buffer, amortized over multiple frames (Section 6), while rendering the mesh using the other index buffer.

\subsection{Static structures}

The vertex hierarchy is stored in the VertexTree buffer. For each vertex $v$ we store the indices $v_{t}, v_{u}, v_{t^{*}}$ and $v^{p}$. Vertex $v$ is also associated with the two triangle faces $f_{l}^{v}$ and $f_{r}^{v}$ that are removed from the mesh by the collapse $\mathrm{col}_{v}$. However, we do not keep their indices explicitly with $v$, since they are simply $2 v$ and $2 v+1$. To guarantee consistency on meshes with boundaries, where $\operatorname{col}_{v}$ may remove only one face $f_{l}^{v}$ at the boundary, we duplicate $f_{l}^{v}$ as $f_{r}^{v}$ and insert it into $M^{n}$. Note that this does not incur any additional rendering cost, and requires negligible memory since there are typically only a small fraction of boundary faces. Additionally, we store parameters used for view-dependent refinement of the non-leaf vertices in the RefineCriteria buffer.

The OrigFaces buffer stores for each face in the original mesh $M^{n}$ its vertex indices $\left\{v_{0}, v_{1}, v_{2}\right\}$ (i.e. 3 leaves in the vertex hierarchy). These indices are normally stored as 32-bit integers to support large models, so each face in OrigFaces would require 12 bytes. However, since meshes with more than $2^{24}$ vertices $(33.5 \mathrm{M}$ triangles) would occupy more than the $1 \mathrm{~GB}$ of available video memory,

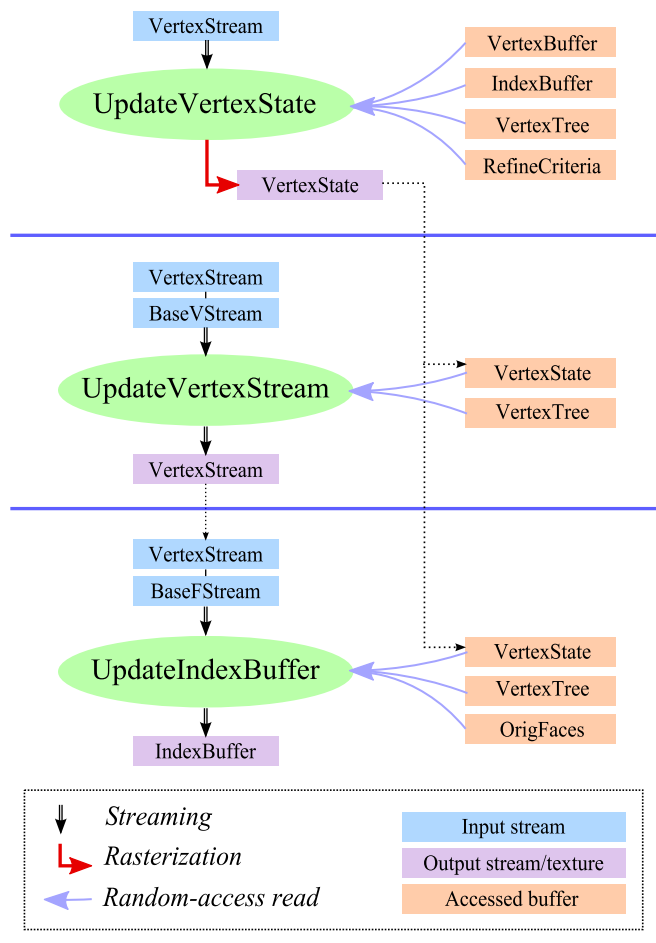

Figure 3: The 3-step selective refinement algorithm.

our implementation uses 24 bits per index ( 9 bytes per face), and uses the saved 3 bytes per face to encode the indices $v_{\operatorname{lmax}}$ and $v_{\text {rmax }}$ from Section 3.2.

Buffer BaseFStream stores the indices of the faces in the base mesh $M^{0}$. And, the indices of the vertices in $M^{0}$ (i.e. the root vertices in the hierarchy) are stored in a buffer BaseVStream to better parallelize the update of VertexStream, as described in Section 5.

\subsection{Dynamic structures}

The VertexState texture stores for each vertex in the hierarchy one of three possible states: $\{$ collapsed, split, splitting $\}$. As their names suggests, the split and collapse states identify vertices that have or have not been split, respectively. The state splitting is needed by the algorithm to identify vertices that are currently being split. For details, refer to Section 5. Note that all vertices in the hierarchy that are above the frontier are in either split or splitting state, while the others are in collapsed state, as shown in Figure 2. We do not have to associate states with the leaves since they cannot split. Due to GPU hardware constraints, we store VertexState as a 2D texture instead of a buffer.

Finally, the main data structure of our algorithm is the VertexStream buffer, which contains a dynamically updated list of all the vertices that have been split (i.e., those that are above the frontier). This buffer is useful for identifying both the active vertices and active faces in the current selectively refined mesh. Specifically the set of active vertices (i.e., those on the frontier and in collapsed state) and the set of active faces are defined as follows:

$$
\begin{aligned}
& \bigcup_{v \in M} v=\bigcup_{v \in \text { VertexStream }} \bigcup_{x \in\left\{v_{t}, v_{u}\right\}}\left\{x \mid x_{\text {state }}=\text { collapsed }\right\}, \\
& \bigcup_{f \in M} f=\text { BaseFStream } \cup\left(\bigcup_{v \in \text { VertexStream }}\left\{f_{l}^{v}, f_{r}^{v}\right\}\right) .
\end{aligned}
$$

Note that we keep two VertexStream buffers, and ping-pong between them to avoid read-modify-write hazards (Section 5). 


\section{Runtime algorithm}

The runtime algorithm dynamically updates IndexBuffer, which stores the triangles in the selectively refined mesh. The update consists of three processing steps as outlined in Figure 3. The first step checks for desirable edge collapses and vertex splits, and updates the vertex states accordingly; the second step updates and maintains the stream of active vertices based on the updated states; and the final step generates the index buffer using the set of split vertices and the updated frontier implied from the states. Next, we describe each of the steps. Listing 1 provides detailed pseudocode.

UpdateVertexState We check for splits and collapses according to the three view-dependent criteria - view frustum, surface orientation and screen-space geometric error - from Hoppe [1998]. Additional splits may be required to enforce the dependency rules. The algorithm starts by first setting the states of all elements in VertexStream to collapsed. Next, it traverses the vertices in VertexStream to update their states. This is accomplished by generating point primitives in the geometry shader and rendering the updated state to the VertexState texture, which is addressable by vertex index. In the traversal, we set the state of the vertices that do not collapse to split. For the children of the vertices in VertexStream that are active in $M$ (following (1)), we test if they should split, and if so, update their states to splitting. In addition, we also test their children. By doing so, we allow up to two hierarchy levels to split simultaneously within each update iteration, thereby resulting in a more efficient update.

Satisfying all split dependencies for the new splits would require performing recursive updates by forcing splits on potentially remote vertices in the mesh. Such splits can be arbitrary in number and in nesting within the hierarchy. Hoppe [1997] makes use of a stack to record and then force the chain of required splits. Unfortunately, such an approach is infeasible on the GPU due to the limitations on the output size of a geometry shader instance. A naïve approach would be to mark dependent splits and wait multiple iterations until all dependencies are satisfied before splitting a vertex. However, this would cause a significant temporal lag in LOD refinement.

To overcome this problem we introduced a cascaded update method that updates new splits without respecting their dependencies, and forces the adjacent constraining vertices to split in subsequent updates. Specifically for each $s p l_{v}$, we continue splitting the active side vertices $v_{l}$ and $v_{r}$ in $f_{l}^{v}$ and $f_{r}^{v}$ every iteration as long as they still constrain $s p l_{v}$. This scheme is significantly faster as it takes many fewer algorithm iterations to fully propagate. The drawback is that it may result in temporary foldovers in the mesh surface. However, the LOD update is so fast that such foldovers are hardly observed in all our experiments (see accompanying video).

Forcing adjacent splits may be in conflict with desired collapses on the same vertices, and it is impossible to explicitly identify these conflicts in one pass since they arise in parallel execution paths. We resolve these conflicts by performing additional compensating splits. Specifically, for the side vertices $v_{l}$ and $v_{r}$ that are involved in the dependent splits from $v$, we also update the states of their parents $\left(v_{l}\right)^{p}$ and $\left(v_{r}\right)^{p}$ to split, in case the parents are collapsing in their own executing instances.

UpdateVertexStream The second step outputs an updated VertexStream by using the stream out functionality. In this step, the algorithm traverses the vertices in VertexStream and outputs all child vertices that are not in collapsed state. We output the children rather than the vertices themselves to save one state check per instance. We output the root vertices in BaseVStream directly in a quick preceding pass. Newly split vertices that are two levels deeper in the hierarchy are identified and output as well.

UpdateIndexBuffer Finally, we update IndexBuffer by writing out the active triangle faces of $M$ (following (2)). More specifically, the indices of the active vertices in every pair of faces $\left\{f_{l}^{v}, f_{r}^{v}\right\}$ as-

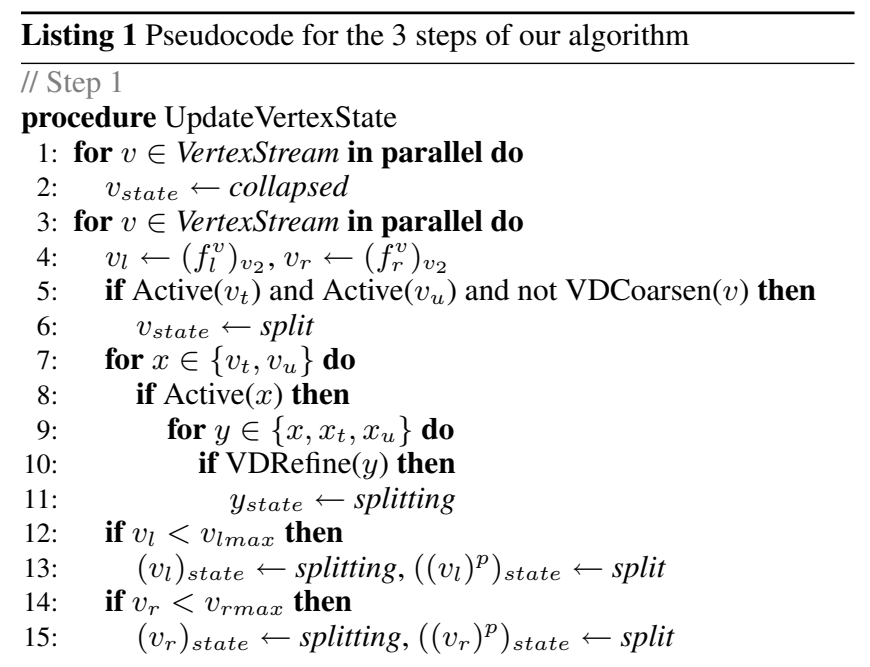

function bool $\operatorname{Active}(v)$

1: // return $v_{\text {state }}=$ collapsed // leads to read-write hazard

2: return $v=\left(v^{p}\right)_{t} \quad ? \quad v=\left(f_{l}^{v^{p}}\right)_{v_{0}} \quad: \quad v=\left(f_{l}^{v^{p}}\right)_{v_{1}}$

function bool VDRefine $(v)$

1: Test view-dep. refinement criteria using $\left\{\delta_{v}, r_{v}, \sin ^{2} \alpha_{v}\right\}$.

function bool VDCoarsen $(v)$

1: return $\left(v_{l}\right)^{p}<v$ and $\left(v_{r}\right)^{p}<v$ and not $\operatorname{VDRefine}(v)$

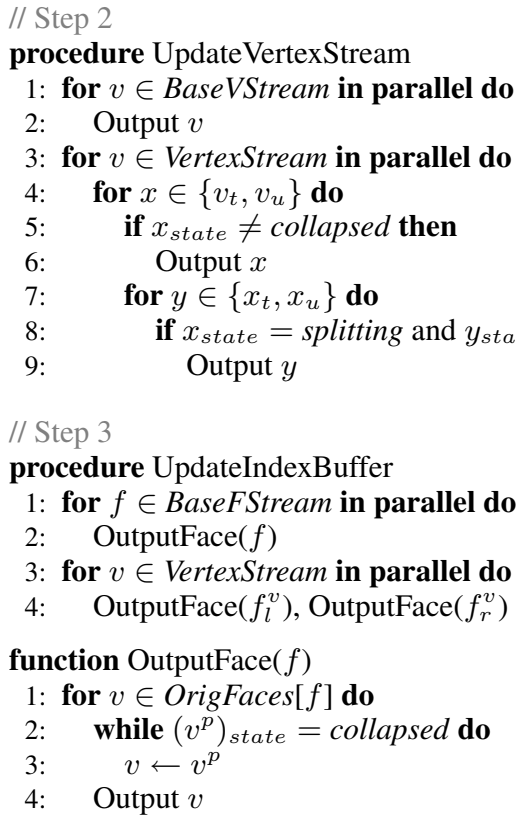

sociated with each split vertex $v$ in the updated VertexStream are streamed out to IndexBuffer. We obtain the indices of the active vertices in $M$ by retrieving the indices of the leaf vertices in the same faces in $M^{n}$ from OrigFaces, and searching up the hierarchy for the coarsest vertices in collapsed states. An additional preceding pass streams out the triangle faces in $M^{0}$ by traversing BaseFStream.

\section{Amortized computation}

Geometry LOD algorithms often amortize the refinement computation over multiple frames to maintain a desired framerate [Hoppe 1997]. Our runtime update algorithm involves three update steps that require different amounts of time. A naïve amortization that performs one update step per frame leads to oscillations in frame times (see Figure 4). To try to maintain an upper bound $T^{*}$ on the 


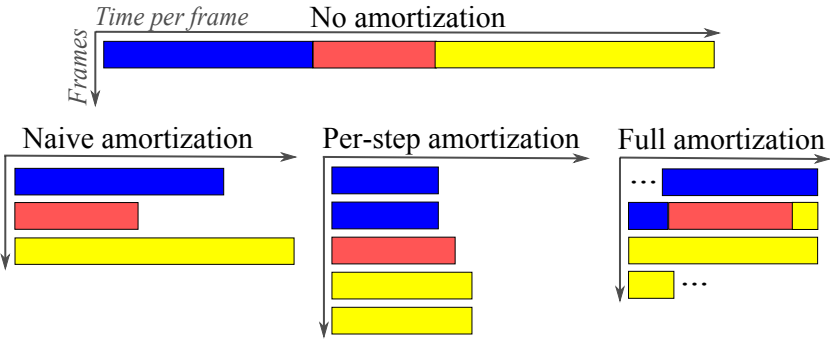

Figure 4: Amortization mechanisms. The width of each bar represents the time incurred by that step of the update algorithm. Full amortization manages to keep frame times low and consistent.

Table 2: Statistics for the original meshes used in our experiments and the number of faces used in the selectively refined meshes shown in Figure 7.

\begin{tabular}{lcr|rr}
\hline \multicolumn{3}{c|}{ Input meshes } & \multicolumn{2}{c}{ Meshes in Figure 7 } \\
\hline $\begin{array}{c}\text { Model } \\
\text { name }\end{array}$ & $\begin{array}{c}\text { Total } \\
\text { \#faces }\end{array}$ & $\begin{array}{c}\text { Memory } \\
(\mathrm{MB})\end{array}$ & $\begin{array}{c}\text { Rendered } \\
\text { \#faces }\end{array}$ & $\begin{array}{c}\text { Rendering } \\
\text { time }(\mathrm{ms})\end{array}$ \\
\hline Lucy & $2,000,000$ & 65.8 & 88,432 & 4.6 \\
Terrain & $2,097,147$ & 69.0 & 179,013 & 7.5 \\
Dragon & $7,218,906$ & 237.5 & 290,892 & 15.2 \\
Statue & $10,000,000$ & 329.0 & 389,566 & 22.2 \\
\hline
\end{tabular}

frame time (e.g., $33.3 \mathrm{~ms}$ ), at the beginning of each update iteration, we dynamically partition the update steps. We explore two such mechanisms.

Per-step amortization The first mechanism partitions each step $i \in\{1,2,3\}$ individually. Specifically, at algorithm iteration $k$, it partitions the input stream $S_{i}^{k}$ for step $i$ uniformly into $N_{i}^{k}$ segments (see Figure 4). The number of segments $N_{i}^{k}$ is estimated based on the previous iteration as $N_{i}^{k}=\left\lceil\frac{\left|S_{i}^{k}\right|}{\left|S_{i}^{k-1}\right|} \cdot \frac{T_{i}^{k-1}}{T^{*}}\right\rceil$ where $T_{i}^{k-1}$ is the total time spent in step $i$ in the previous iteration. The mechanism automatically adapts to the update load of the selectively refined mesh at runtime, but still suffers from frame time oscillations due to the absence of load balancing across different steps of the algorithm.

Full amortization The second mechanism alleviates this problem by allowing multiple algorithm steps to be executed within the same frame (see Figure 4). As in the previous approach, this method also keeps an estimate of the cost of each step based on the previous update. The algorithm determines what fraction of the current step can be executed within the current frame time budget $T^{*}$. If the step can only be executed partially, the remainder is carried over to the following frame. If the step can be executed fully, then the algorithm again determines what fraction of the upcoming step fits within the remaining budget and can be executed. The algorithm proceeds in this fashion until it exhausts the time budget.

\section{Results}

We implemented our algorithm in Microsoft DirectX 10 using an Intel Core 2 CPU with 2GB memory and an NVIDIA GeForce 8800 GTX graphics card. All shaders are written in HLSL using shader model 4.0. For all examples we use a window size of 1280x800 pixels and a screen-space error tolerance of 1 pixel.

Frame rate Figure 7 shows selectively refined meshes for the models in Table 2. The frame times for the renderings in Figure 7a are shown in Table 2.

Figure 5 graphs the relationship between the number of faces in the current active mesh $M$ and the time it takes to perform all of

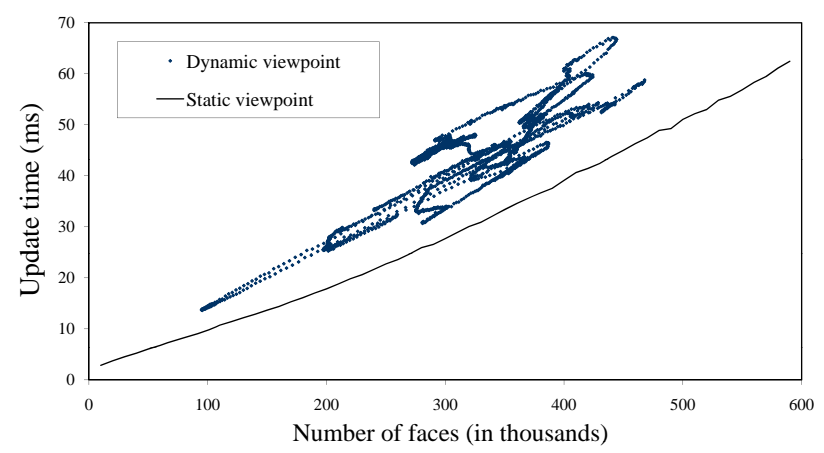

Figure 5: Update times as a function of the number of faces in the active mesh $M$ when no splits and collapses are necessary (static viewpoint), and when splits and collapses are applied as part of typical viewer motion (dynamic viewpoint).
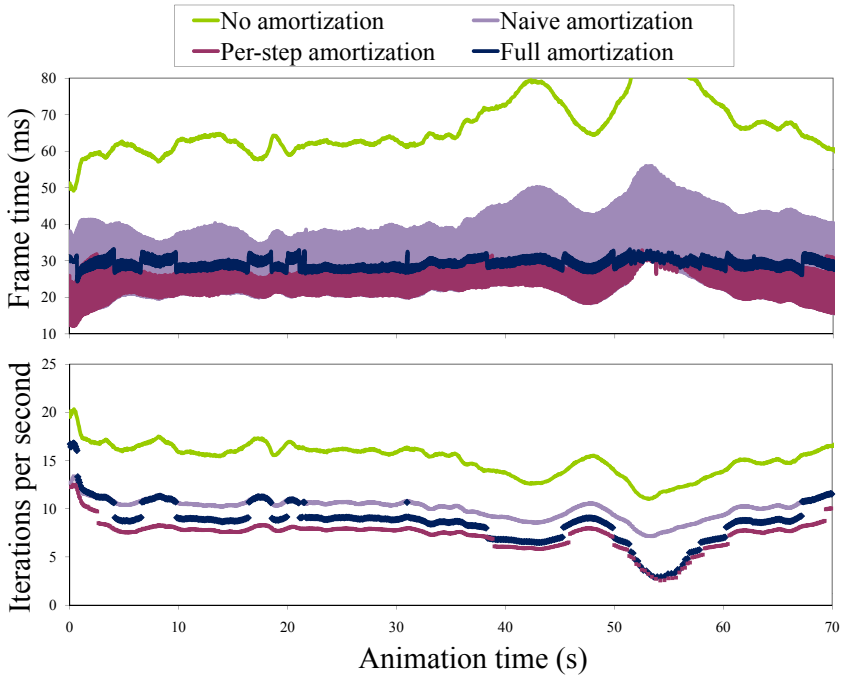

Figure 6: Timings measurements (top) and algorithm iterations per second (bottom) for the Statue rendering sequence using different amortization strategies.

the algorithm passes. With a static viewpoint, the processing time is clearly linear on $M$. This linear-time computation has been observed for all of the models in our test suite. Figure 5 also graphs the processing time when using a typical camera motion (dynamic viewpoint). Note that the introduced splits and collapses cause additional processing and geometry amplification, thus slowing down the update. The added GPU data amplification cost is not prohibitively expensive and the relationship remains roughly linear on the size of $M$. The variation is due to the differing number of splits and collapses for the measurements.

Figure 6 (top) graphs the frame time of each amortization method for a Statue rendering sequence using a target frame time upper bound $T^{*}$ of $33.3 \mathrm{~ms}$. Without amortization, the cost incurred by the update is clearly above the desired frame time. Naïve amortization partitions the update into 3 frames thereby improving frame time significantly. However, due to the high relative costs among the update passes, the frame time still oscillates heavily. Per-step amortization further partitions each step to ensure that frame times are no higher than $T^{*}$. Note, that, while it reduces frame times, varying step costs may still cause significant oscillation. Finally, the full amortization method is able to better maintain the target frame rate by allowing multiple passes to be processed within the same frame, as described in Section 6. Note, however, that a completely stable frame time cannot be guaranteed since the GPU al- 
Table 3: Comparison of memory size with prior schemes.

\begin{tabular}{ll}
\hline \multicolumn{1}{c}{ View-dependent LOD scheme } & Memory size (bytes) \\
\hline VDPM [Hoppe 1997] & $216 n$ \\
SVDLOD [Hoppe 1998] & $88 n+100 m$ \\
MT [Floriani et al. 1998] & $75 n$ \\
VDT [El-Sana and Varshney 1999] & $90 n$ \\
FastMesh [Pajarola and DeCoro 2004] & $88 n+6 m$ \\
Our scheme & $69 n+56 m$ \\
\hline
\end{tabular}

gorithm cannot predict drastic changes in $M$, which would result in immediate slowdowns in some of the passes. Therefore, occasional performance spikes are unavoidable. However, even with the nearly-dizzying camera motions shown in the accompanying video, the spikes are rare and do not significantly harm the observed frame rate across this entire sequence.

Both per-step and full amortization manage to reduce frame time to the desired target bound $T^{*}$. When only considering these two satisfactory options, full amortization manages to accommodate the entire update in fewer frames, as predicted in Figure 4 and evidenced in Figure 6 (bottom), which graphs the total number of iterations per second.

Memory analysis Our data structures use a total of $69 n+56 m$ bytes, where $n$ and $m$ are the numbers of vertices in the original and active meshes respectively. For sufficiently detailed models, it is generally impossible to view all surface regions at high resolution within a frame, so typically $m \ll n$. Thus the memory bottleneck is the size $69 n$ of the static portion of our structures. As shown in Table 3, this compares favorably with previous viewdependent LOD schemes, which is surprising given our challenging parallelism restrictions. In addition, most prior schemes perform immediate-mode rendering (e.g. with glVertex() calls), whereas our dynamic data structures ( $56 \mathrm{~m}$ bytes) maintain explicit index buffers that permit more efficient rendering on present graphics systems.

Limitations Our index buffers define indexed triangle lists rather than strips, so take more space than an optimized static mesh. Also, the ordering of faces within this list is not optimized for vertex cache locality. Finally, our system does not yet support geomorphs for smooth temporal transitions. However, the LOD updates are fast and the screen-space error tolerance is small enough that popping is nearly imperceptible.

\section{Conclusion and future work}

We present the first view-dependent LOD algorithm for vertex-level mesh refinement that operates entirely on the GPU. Our scheme performs general vertex splits and edge collapses to incrementally and selectively refine an irregular hierarchy as a sequence of parallel streaming steps. The approach is highly parallel, processing many splits and collapses simultaneously. Because the mesh is updated and rendered using a constant number of draw calls, CPU utilization is near zero. The cost of these draw calls is further amortized over multiple frames using a simple feedback mechanism.

One of our contributions is to overcome the difficulties imposed by GPU parallel processing. This involves a new, compact dependency structure and a cascaded update method. In future work, it would be interesting to consider more general parallel processing APIs. For instance, NVIDIA's CUDA and AMD's Stream Computing expose local shared memory and allow scattered reads and writes to the same memory buffer. The current version of CUDA does not support parallel streaming whereby each stream instance can output a variable number of elements, which is essential in our approach. However, it is likely that such APIs will continue to improve in programming flexibility.

The parallel nature of our algorithm lets it scale with the number of stream processors in the GPU. Thus we expect greater perfor- mance gains as the GPU evolves to include more processors. The stream-based approach may also be applicable to other parallel architectures, as it provides an elegant way to decouple an irregular refinement algorithm into dependency-free passes. Finally, we hope that our ideas may inspire new algorithms for handling irregular data structures within parallel architectures.

\section{Acknowledgments}

We would like to thank Lei Yang for help on the video production, and the anonymous reviewers for their comments. The Dragon and Statue are courtesy of the Stanford 3D scanning repository.

\section{References}

BOKELOH, M. and WAND, M. 2006. Hardware accelerated multiresolution geometry synthesis. In I3D '06: Proceedings of the 2006 Symposium on Interactive $3 D$ graphics and games, pages 191-198.

Borgeat, L., Godin, G., Blais, F., Massicotte, P., and LAHANIER, C. 2005. GoLD: interactive display of huge colored and textured models. ACM Transactions on Graphics, 24(3): 869-877.

Boubekeur, T. and Schlick, C. 2005. Generic mesh refinement on GPU. In HWWS '05: Proceedings of the ACM SIGGRAPH/Eurographics conference on Graphics hardware, pages 99-104.

Buatois, L., Caumon, G., and LÉvy, B. 2006. GPU accelerated isosurface extraction on tetrahedral grids. International Symposium on Visual Computing, pages 383-392.

Cignoni, P., Ganovelli, F., Gobbetti, E., Marton, F., Ponchio, F., and Scopigno, R. 2003. BDAM: batched dynamic adaptive meshes for high performance terrain visualization. Computer Graphics Forum, 22:505-514.

Cignoni, P., Ganovelli, F., Gobbetti, E., Marton, F., PonCHIO, F., and ScOPIGNO, R. 2004. Adaptive tetrapuzzles: Efficient out-of-core construction and visualization of gigantic multiresolution polygonal models. ACM Transactions on Graphics, 23(3):796-803.

DeCoro, C. and TAtarchuk, N. 2007. Real-time mesh simplification using the GPU. In I3D '07: Proceedings of the 2007 Symposium on Interactive $3 D$ graphics and games, pages 161166.

Duchaineau, M., Wolinsky, M., Sigeti, D. E., Miller, M. C., Aldrich, C., and MineEv-Weinstein, M. B. 1997. ROAMing terrain: real-time optimally adapting meshes. In IEEE Visualization 97, pages 81-88.

El-SAnA, J. and VARShney, A. 1999. Generalized viewdependent simplification. In Proceedings of Eurographics 99, pages $83-94$.

Floriani, L. D., Magillo, P., and Puppo, E. 1998. Efficient implementation of multi-triangulations. In VIS '98: Proceedings of the conference on Visualization '98, pages 43-50.

Guthe, M., BALÁzs, A., and Klein, R. 2005. GPU-based trimming and tessellation of nurbs and T-spline surfaces. ACM Transactions on Graphics, 24(3):1016-1023.

Hoppe, H. 1997. View-dependent refinement of progressive meshes. In Proceedings of ACM SIGGRAPH 1997, pages 189198.

Hoppe, H. 1998. Smooth view-dependent level-of-detail control and its application to terrain rendering. In VIS '98: Proceedings of the conference on Visualization '98, pages 35-42.

JI, J., WU, E., LI, S., and LIU, X. 2006. View-dependent refinement of multiresolution meshes using programmable graphics hardware. The Visual Computer, 22(6):424-433. 

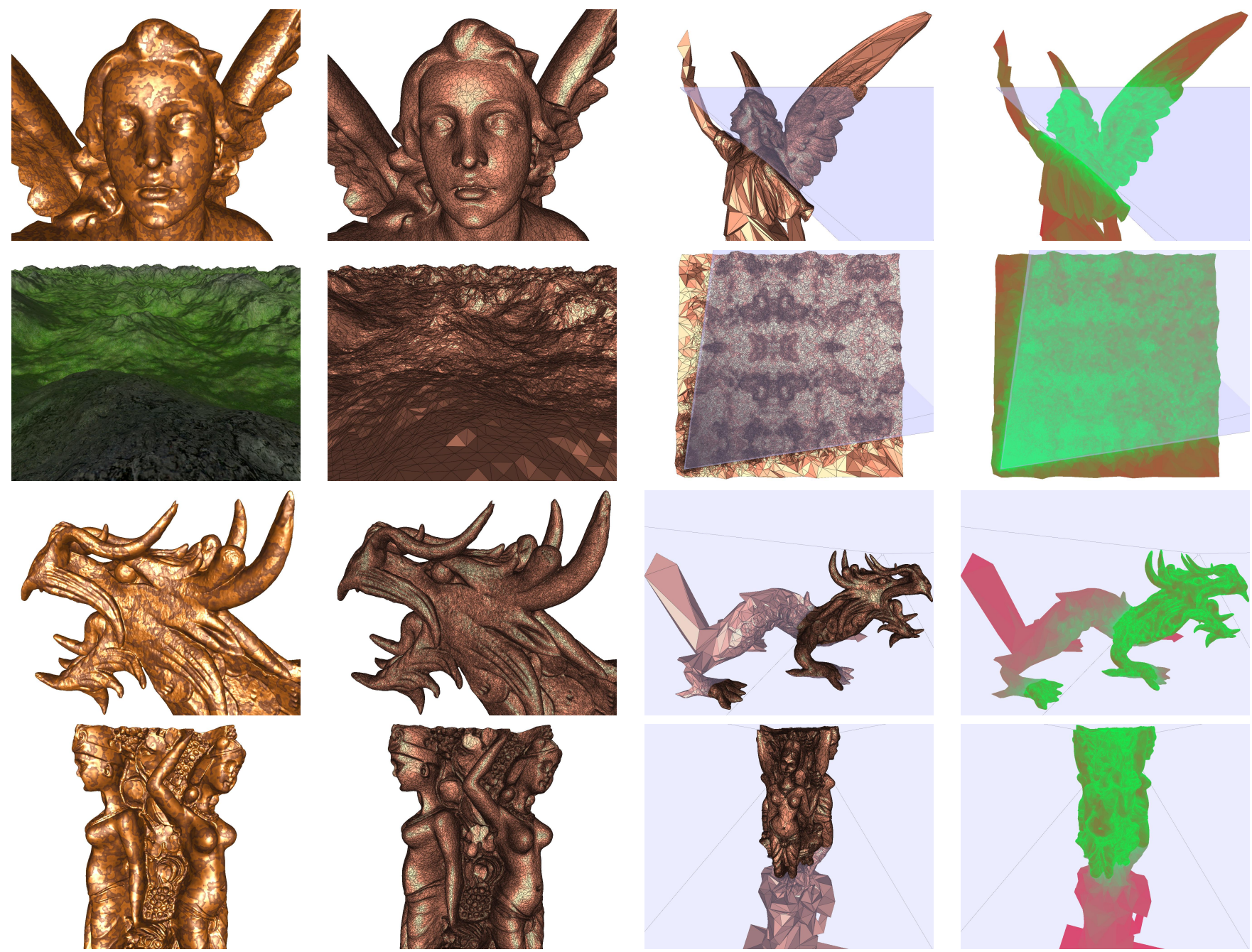

(a)

(b)

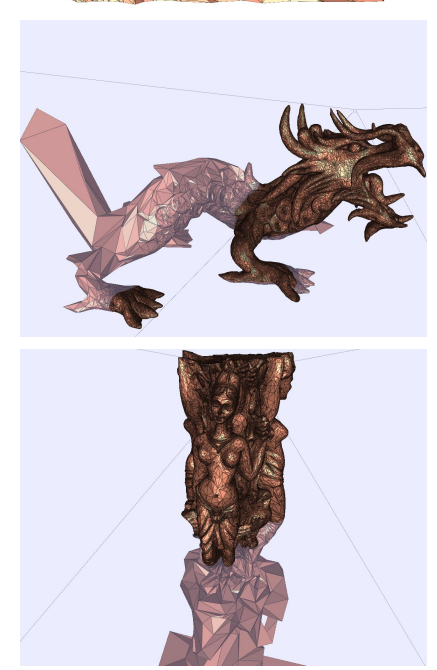

(c)

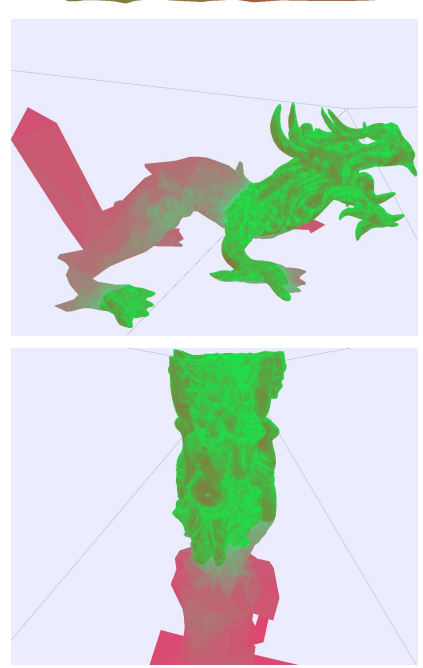

(d)

Figure 7: Renderings of our selectively refined meshes with a procedural texture (a) and in wireframe mode (b). The same mesh is then visualized from a different viewpoint in wireframe mode $(c)$, and with color-coded $L O D(d)$, where green represents higher detail than red. Note that mesh regions that lie outside the frustum, farther away, or facing away from the camera, have lower LODs. The error tolerance is less than one pixel.

Kobbelt, L., Campagna, S., and Peter Seidel, H. 1998. A general framework for mesh decimation. In Proceedings of Graphics Interface, pages 43-50.

LEVENBERG, J. 2002. Fast view-dependent level-of-detail rendering using cached geometry. In VIS '02: Proceedings of the conference on Visualization '02, pages 259-266.

Lindstrom, P., Koller, D., Ribarsky, W., Hodges, L. F., FAust, N., and Turner, G. A. 1996. Real-time, continuous level of detail rendering of height fields. In Proceedings of ACM SIGGRAPH 1996, pages 109-118.

Lindstrom, P. and PASCUCCI, V. 2002. Terrain simplification simplified: A general framework for view-dependent out-of-core visualization. IEEE TVCG, 8(3):239-254.

LosASSO, F. and Hoppe, H. 2004. Geometry clipmaps: terrain rendering using nested regular grids. ACM Transactions on Graphics, 23(3):769-776.

LUEBKE, D. and ERIKSON, C. 1997. View-dependent simplification of arbitrary polygonal environments. In Proceedings of ACM SIGGRAPH 1997, pages 199-208.

LuebKe, D., Watson, B., Cohen, J. D., Reddy, M., and
VARSHNEY, A. 2002. Level of Detail for 3D Graphics. Elsevier Science Inc., New York, NY, USA.

PAjarola, R. and DeCoro, C. 2004. Efficient implementation of real-time view-dependent multiresolution meshing. IEEE TVCG, 10(3):353-368.

PASCUCCI, V. 2004. Isosurface computation made simple: hardware acceleration, adaptive refinement and tetrahedral stripping. In Joint Eurographics - IEEE TVCG Symposium on Visualization (VisSym), pages 293-300.

SAnder, P. V. and Mitchell, J. L. 2005. Progressive buffers: View-dependent geometry and texture for LOD rendering. In SGP '05: Proceedings of the third Eurographics Symposium on Geometry Processing, pages 129-138.

Shiue, L.-J., Jones, I., and Peters, J. 2005. A realtime GPU subdivision kernel. ACM Transactions on Graphics, 24(3):10101015.

Xia, J. C. and Varshney, A. 1996. Dynamic view-dependent simplification for polygonal models. In VIS '96: Proceedings of the 7th conference on Visualization '96, pages 327-334. 


\section{Appendix}

Lemma 1. The dependency rules (I) and (II) conform to (i) and (ii) respectively, i.e., $(i) \Rightarrow(I)$ and $(i i) \Rightarrow(I I)$.

Proof. (i) $\Rightarrow(\mathrm{I})$ : Suppose $v_{l}>v_{l \max }$. We first prove that $f_{n_{0}}^{v}$ exists. By definition of $v_{l \max }, v_{l} \geq c_{v}\left(f_{n_{0}}^{v}\right)$ and $v_{l} \geq c_{v}\left(f_{n_{1}}^{v}\right)$. We denote $x=c_{v}\left(f_{n_{0}}^{v}\right)$. The face $f_{n_{0}}^{v}$ exists in the current selectively refined mesh if $x=0$ by definition, otherwise $x \neq 0$ and $f_{n_{0}}^{v}$ is created by $s p l_{x}$, which means the vertex $x$ is an ancestor of two vertices in triangle $f_{n_{0}}^{v}$. Since $x \neq 0, x$ is not an ancestor of $v$ (and therefore $v_{t}$ ). And because $v_{l}$ is adjacent to $f_{n_{0}}^{v}$ and $v_{l} \geq x, x$ must be an ancestor of $v_{l}$. Therefore $f_{n_{0}}^{v}$ also exists since $s p l_{x}$ has been performed otherwise $v_{l}$ would not be active. The same argument holds for the existence of $f_{n_{1}}^{v}$, and similarly supposing $v_{r}>v_{r \max }$, then $\left\{f_{n_{2}}^{v}, f_{n_{3}}^{v}\right\}$ also exist. Since (i) is true, it follows that whenever $v_{l}>v_{l \max }$ and $v_{r}>v_{r \max }$, then $\left\{f_{n_{0}}^{v}, f_{n_{1}}^{v}, f_{n_{2}}^{v}, f_{n_{3}}^{v}\right\}$ all exist, and $s p l_{v}$ is legal, i.e. (I) is true.

(ii) $\Rightarrow\left(\right.$ II): Since $v_{t}$ and $v_{u}$ are active, $s p l_{v}$ has been legally performed. According to Hoppe [1997], $\left\{f_{n_{0}}^{v}, f_{n_{1}}^{v}\right\}$ and $\left\{f_{n_{2}}^{v}, f_{n_{3}}^{v}\right\}$ must be pairwise adjacent at the time of $s p l_{v}$. Now suppose $\left(v_{l}\right)^{p}<v$. We first prove that $f_{l}^{v}$ is adjacent to $f_{n_{0}}^{v}$. We denote $f^{\prime}$ as the face adjacent to $v_{t}, v_{l}$ and $f_{l}$, and denote $x^{\prime}$ as the vertex such that $s p l_{x^{\prime}}$ creates $f^{\prime}$. Suppose $f^{\prime} \neq f_{n_{0}}^{v}$. $x^{\prime}$ cannot be an ancestor of $v_{t}$ since $f_{n_{0}}^{v}$ and $f_{n_{1}}^{v}$ would otherwise not be adjacent when performing $s p l_{v}$. Then $x^{\prime}$ must be an ancestor of $v_{l}$ because $s p l_{x^{\prime}}$ creates $f^{\prime}$ and $x^{\prime}$ is an ancestor of two vertices in $f^{\prime}$. Since $f^{\prime}$ is adjacent to $f_{l}, s p l_{x^{\prime}}$ must be performed after $s p l_{v}$ to be considered legal, i.e. $x^{\prime}>v$, since otherwise $f_{l}$ would not be present at the time of $s p l_{x^{\prime}}$. However as $x^{\prime}$ is an ancestor of $v_{l}$, $x^{\prime} \leq\left(v_{l}\right)^{p}<v$, which is a contradiction. Therefore $f^{\prime}=f_{n_{0}}^{v}$ and $f_{l}^{v}$ is adjacent to $f_{n_{0}}^{v}$. By the same argument, it can be shown that $f_{l}^{v}$ is also adjacent to $f_{n_{1}}^{v}$, and similarly supposing $\left(v_{r}\right)^{p}<v$, then $f_{r}^{v}$ is adjacent to $\left\{f_{n_{2}}^{v}, f_{n_{3}}^{v}\right\}$. Since (ii) is true, it follows that if $\left(v_{l}\right)^{p}<v$ and $\left(v_{r}\right)^{p}<v$, then $f_{l}^{v}$ is adjacent to $\left\{f_{n_{0}}^{v}, f_{n_{1}}^{v}\right\}$ and $f_{r}^{v}$ is adjacent to $\left\{f_{n_{2}}^{v}, f_{n_{3}}^{v}\right\}$. As a result, colv is legal, i.e. (II) is also true. 